\title{
Asteroid Hyalosis: A Mimic of Vitreous Hemorrhage on Point of Care Ultrasound
}

\author{
Charles E.A. Stringer, MSc, MD*; Justin S. Ahn, $\mathrm{MD}^{*^{\dagger}}$; Daniel J. Kim, MD*
}

\section{ABSTRACT}

Point of care ultrasound in the emergency department (ED) is increasingly being used to diagnose time-sensitive, vision-threatening conditions. We present a case of a 64-year-old female who presented to the ED with a three-day history of worsening left eye floaters. Point of care ocular ultrasound demonstrated a posterior chamber containing many echogenic opacities of varying size without acoustic shadowing. Movement of the eye resulted in significant after-movement of these opacities, giving the classic "washing machine" appearance seen with vitreous hemorrhage (VH). Based on these ultrasound findings, the patient was diagnosed with a $\mathrm{VH}$ and was referred to ophthalmology. The consulting ophthalmologist ultimately diagnosed the patient with asteroid hyalosis without VH. Asteroid hyalosis is a benign condition of the vitreous resulting in calcium phosphate and lipid deposits that can mimic more serious $\mathrm{VH}$ on point of care ultrasound. Knowledge of this mimic is helpful for communication with specialists and for awareness of the potential for misdiagnosis with ocular ultrasound.

\section{RÉSUMÉ}

On a de plus en plus recours, au service des urgences (SU), à l'échographie au point de service (EPS) pour diagnostiquer des affections dans lesquelles le temps compte et qui sont susceptibles d'altérer sérieusement la vision. Sera exposé ici le cas d'une femme de 64 ans, venue consulter au SU pour la présence croissante, depuis trois jours, de corps flottants dans l'œil gauche. L'EPS de l'œil a révélé la présence de nombreuses opacités échogènes, de taille variable, sans ombre acoustique, dans la chambre postérieure. Les mouvements de l'œil étaient suivis d'un déplacement important de ces opacités, qui ressemblait à l'image classique de la « machine à laver », observée dans les hémorragies vitréennes (HV). Compte tenu des résultats de l'examen, un diagnostic d'HV a été posé, et la patiente a été dirigée vers le service d'ophthalmologie. L'ophthalmologiste consultant a fini par diagnostiquer une hyalite astéroïde sans HV. L'hyalite astéroïde est une affection bénigne du corps vitré qui entraîne des dépôts de phosphate de calcium et de lipides, susceptibles de simuler à l'EPS des images d'HV, une affection plus grave que l'HA. Le fait de connaître l'existence de cette possible confusion facilite les communications avec les spécialistes et sensibilise les médecins au risque de diagnostic erroné, posé à la suite d'un examen par échographie de l'œil.

Keywords: asteroid hyalosis, vitreous hemorrhage, posterior vitreous detachment, retinal detachment, ultrasound

\section{CASE REPORT}

A 64-year-old female presented to the emergency department (ED) with a three-day history of worsening left eye floaters. The floaters had evolved from involvement of a small area of her central vision to the entire visual field of her left eye. She had associated eye irritation. There was no history of flashing lights, visual field loss, discharge, redness, diplopia, or headache. She denied any history of trauma. She had occasional floaters starting two years prior and was diagnosed with bilateral posterior vitreous detachments (PVD) and right eye retinal hemorrhage at that time. The patient had no history of eye surgery or other ocular disease. She did not require the use of glasses or contact lenses

From the *Department of Emergency Medicine, University of British Columbia, Vancouver, BC; tDepartment of Emergency Medicine, Royal Columbian Hospital, New Westminster, BC; and the ¥Department of Emergency Medicine, Vancouver General Hospital, Vancouver, BC.

Correspondence to: Daniel Kim, Department of Emergency Medicine, Vancouver General Hospital, 855 12th Ave W, Vancouver, BC V5Z 1M9, Canada; Email: dkim000@gmail.com 
for visual correction. Past medical history was only significant for gout. She had no history of diabetes, hypertension, or dyslipidemia.

On physical examination, the patient presented with normal vital signs including blood pressure of $119 / 82 \mathrm{~mm} \mathrm{Hg}$. Visual acuity was 20/20 OD and 20/40 OS without the use of a pinhole. Pupils were equal and reactive to light with no relative afferent pupillary defect. There was no redness or conjunctivitis. Extraocular movements were full, and no visual field deficits were appreciated. Intraocular pressures were $15 \mathrm{~mm} \mathrm{Hg}$ bilaterally, and there was no fluorescein uptake on slit lamp examination.

Point of care ultrasound of the left eye demonstrated a posterior chamber containing many echogenic opacities of varying size without acoustic shadowing (Figure 1). Movement of the eye along the long axis of the ultrasound probe revealed significant aftermovement of the heterogeneous echogenic opacities within the vitreous humor, giving a "washing machine" appearance (Video 1). The scan was interpreted as vitreous hemorrhage $(\mathrm{VH})$ without retinal detachment (RD). Accordingly, the patient was referred to the ophthalmology clinic. The ophthalmologist assessed the patient later that day. Visual acuity was 20/20 OD and 20/25 OS under ideal conditions using a pinhole to correct refractive error. Indirect ophthalmoscopy revealed asteroid hyalosis in the left eye. There was no evidence of $\mathrm{VH}$ or RD. No therapy was required, and subsequent follow-up was uneventful.

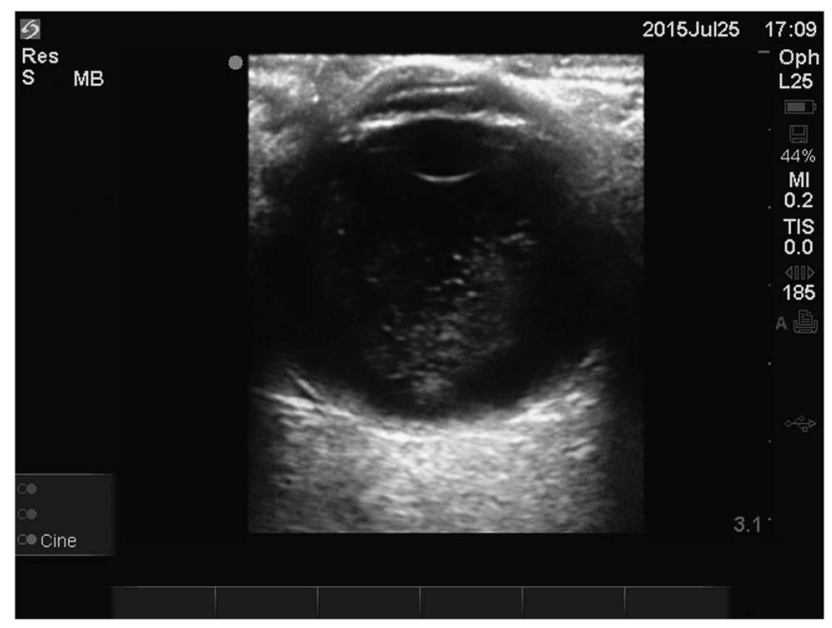

Figure 1. Transverse view of the left eye demonstrates echogenic opacities of varying size without acoustic shadowing in the vitreous humor due to asteroid hyalosis.

\section{DISCUSSION}

Ultrasound has been used for diagnosis in ophthalmology since the 1950s. ${ }^{1}$ More recently, point of care ocular ultrasound is finding a role in the ED for the diagnosis of time-sensitive, vision-threatening conditions such as RD. ${ }^{2}$ Ocular ultrasound is performed by using a linear, high-frequency probe placed over a closed eyelid with copious amounts of gel (or a water balloon) used as an acoustic coupling medium inbetween (Figure 2). The sonographer avoids placing pressure on the globe by bracing the scanning hand against the patient's face (either the bridge of the nose or the zygomatic arch). Ocular presets are recommended, and normal anatomy is confirmed in the transverse plane with the patient's eyes oriented forward. The proper plane is achieved when the lens and optic nerve are in the same view (Figure 3). Fan the transducer through the entire eye to visualize the entire globe. The patient is then asked to look left and right, allowing for a dynamic assessment of the posterior chamber in this plane. The same protocol is then repeated in a longitudinal plane (Figure 4). Comparison with the asymptomatic eye is recommended.

RD and PVD both appear as a linear membrane in the posterior chamber that moves with ocular movements. RD is characterized by a thicker, more echogenic membrane typically attached to the optic nerve (Figure 5, Video 2), whereas PVD appears as a thinner, less echogenic, undulating membrane (Figure 6, Video 3). ${ }^{1,3}$ To visualize $\mathrm{VH}$ by ultrasound,

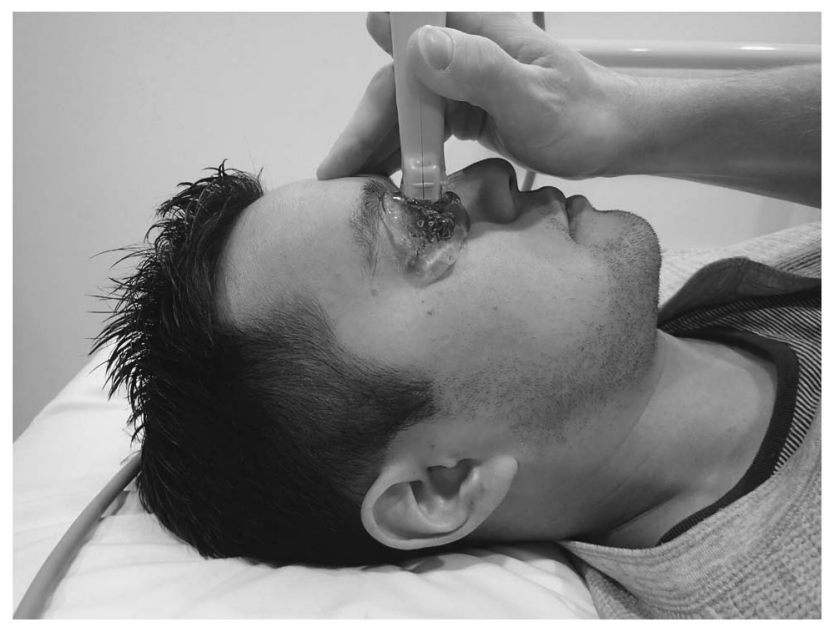

Figure 2. Proper probe position for ocular ultrasound in the transverse plane. Considerable gel is used to keep the probe off the eyelid while gently bracing the hand against the patient's face. 


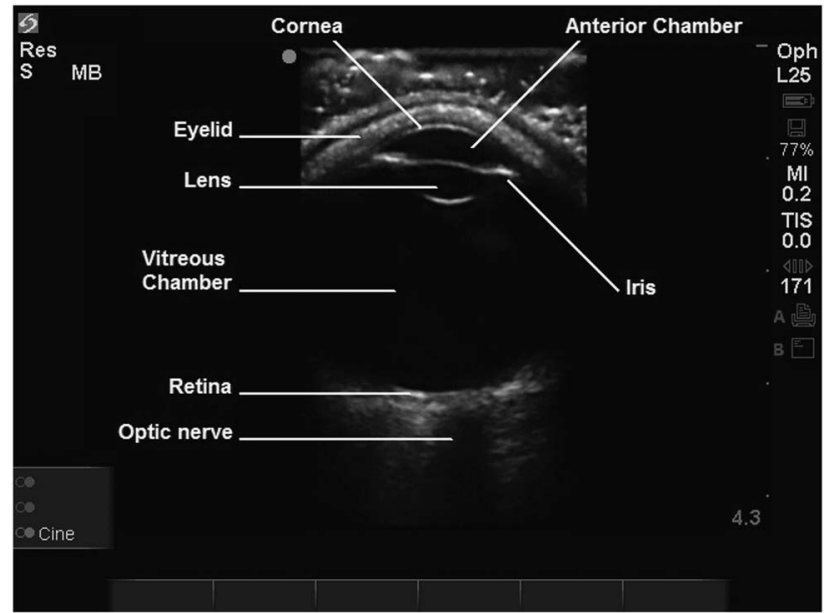

Figure 3. Normal ultrasound anatomy of the eye. In the correct plane, the lens and the optic nerve are both in view.

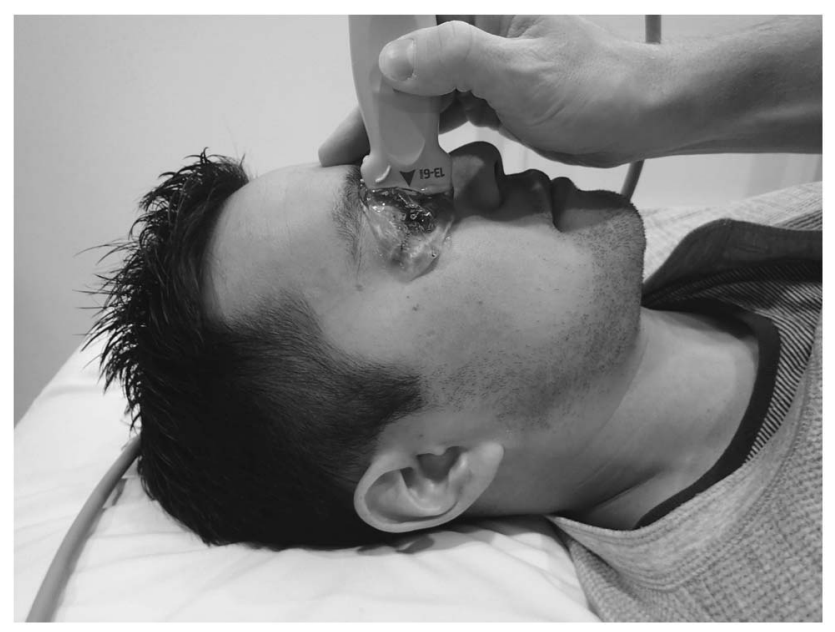

Figure 4. Proper probe position for ocular ultrasound in the longitudinal plane. Considerable gel is used to keep the probe off the eyelid while gently bracing the hand against the patient's face.

the gain should be increased until echogenic material is seen in the posterior chamber. VH looks like multiple heterogeneous echogenic layers that distinctly "tumble" with ocular movement, earning the comparison to a washing machine (Figure 7, Video 4). ${ }^{1,3}$ As ocular ultrasound use becomes more prominent in the ED, it is important to understand when it may lead the operator astray. Accordingly, we report this case of asteroid hyalosis mimicking $\mathrm{VH}$ on ultrasound.

Asteroid hyalosis is a degenerative condition of the vitreous associated with calcium phosphate and lipid deposits. ${ }^{4}$ Large autopsy studies have found a prevalence of $0.83 \%-1.96 \%$. It is associated with increased age, male gender, and PVD. ${ }^{5,6}$ Reported associations

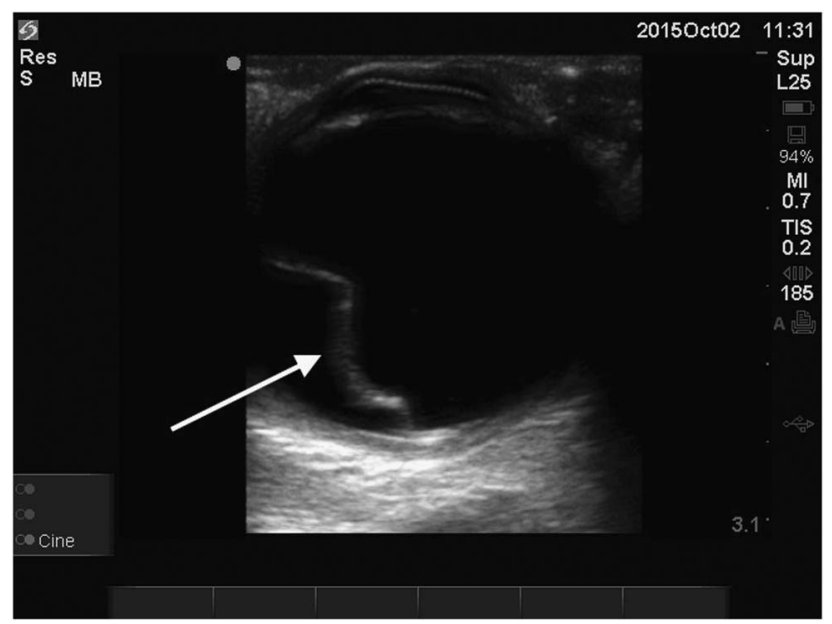

Figure 5. Ultrasound example of retinal detachment, characterized by a thick echogenic membrane in the vitreous chamber (arrow).

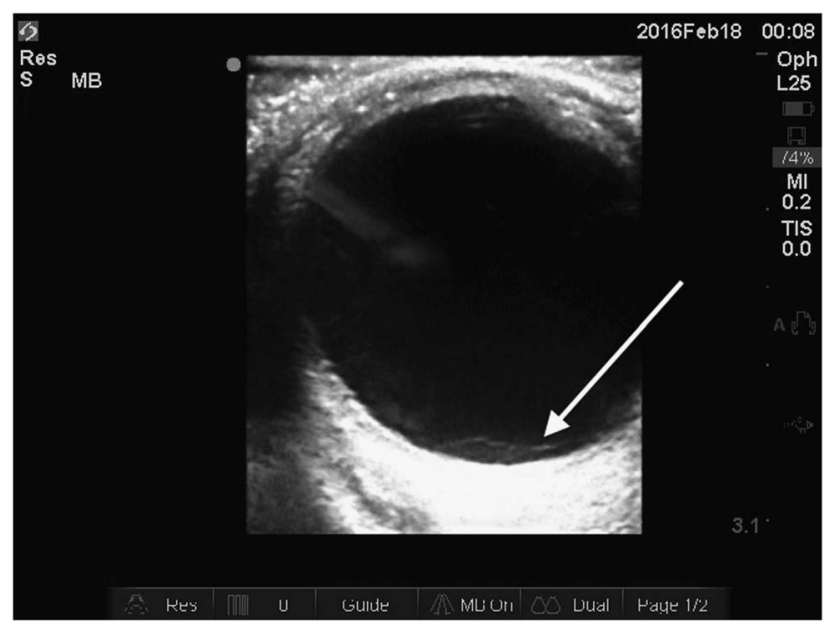

Figure 6. Ultrasound example of posterior vitreous detachment, characterized by a thin, poorly echogenic membrane in the vitreous chamber (arrow).

with hypertension, atherosclerosis, gout, and diabetes are controversial. Asteroid hyalosis is typically unilateral and asymptomatic, although there are case reports of floaters and decreased visual acuity. ${ }^{4,7}$ Ophthalmoscopy reveals multiple yellow-white, iridescent opacifications of varied shape in the vitreous. Accordingly the condition was named for resembling "stars on a clear night." 8 These particles move with eye movement and often obscure the retina. Asteroid hyalosis rarely requires treatment; vitrectomy is reserved for cases with significant vision loss without another etiology. ${ }^{10}$

Sonographically, there is considerable similarity between asteroid hyalosis and VH (Figure 1, Figure 7). Both result in posterior chamber echogenic opacities. 


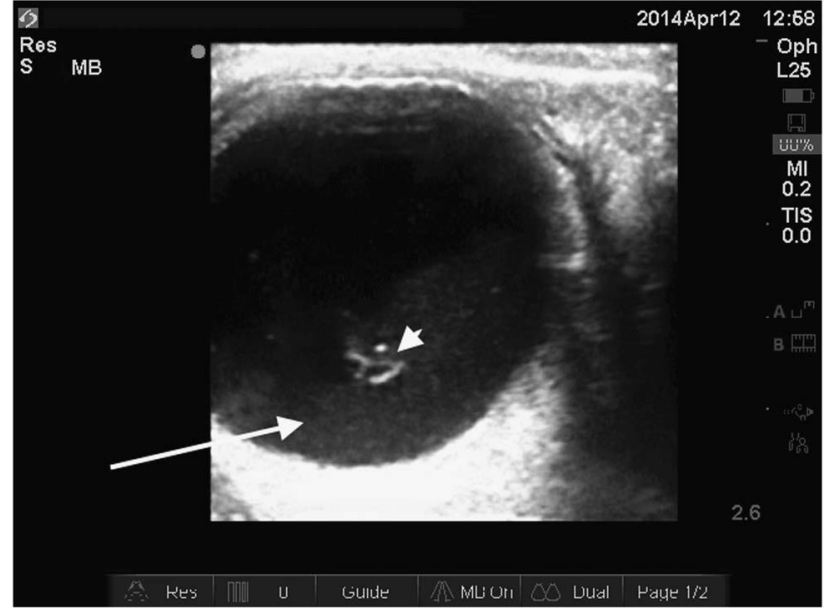

Figure 7. Ultrasound example of vitreous hemorrhage with echogenic blood layered posteriorly in the vitreous chamber (arrow). The overlying hyperechoic material is clotted blood (arrowhead).

The most striking similarity is the after-movement observed with ocular movement. As the eye ceases rotation, the vitreous continues to move causing the echogenicity from asteroid hyalosis and $\mathrm{VH}$ to swirl, resembling clothes tumbling in a washing machine (Video 1, Video 4). ${ }^{1,3}$ The described differences between asteroid hyalosis and $\mathrm{VH}$ are subtle and due to differences in echogenicity. Asteroid hyalosis echoes are usually more discrete echogenic opacities without posterior acoustic shadowing (Figure 1). ${ }^{10} \mathrm{VH}$ echogenicity is described as heterogeneous echogenic layers, although significant hemorrhage can cause poorly-defined, hyperechoic clots (Figure 7). ${ }^{3}$ As the sonographic similarities between asteroid hyalosis and $\mathrm{VH}$ are much more striking than the differences, asteroid hyalosis has considerable potential to be mistaken for $\mathrm{VH}$.

\section{CONCLUSION}

As point of care ocular ultrasound is increasingly used by emergency physicians, awareness of possible ultrasound mimics, such as asteroid hyalosis, is important. Knowledge of asteroid hyalosis may be helpful for communication with specialist colleagues or interpretation of their consultations. However, it is of utmost importance to avoid the misdiagnosis of $\mathrm{VH}$ as asteroid hyalosis. Since asteroid hyalosis is a benign condition, it is important to be cautious and to manage these patients as presumptively having VH by seeking ophthalmologic assessment, especially if the patient is experiencing vision loss.

Competing Interest: None declared.

\section{SUPPLEMENTARY MATERIAL}

To view supplementary material for this article, please visit http://dx.doi.org/doi:10.1017/cem.2016.358

\section{REFERENCES}

1. Chesney GL, Mohabir PK. Ocular ultrasound. In: Point-ofCare Ultrasound (eds. Soni NJ, Arntfield R, Kory P). Philadelphia: Elsevier Saunders; 2015, 245-53.

2. Vrablik ME, Snead GR, Minnigan HJ, et al. The diagnostic accuracy of bedside ocular ultrasonography for the diagnosis of retinal detachment: a systematic review and meta-analysis. Ann Emerg Med 2015;65(2):199-203.e1.

3. Shinar Z, Chan L, Orlinsky M. Use of ocular ultrasound for the evaluation of retinal detachment. 7 Emerg Med 2011; 40(1):53-7.

4. Heagley D, Cantley R, Pitelka L, et al. Asteroid hyalosis of the vitreous humor: an uncommon finding. Diagn Cytopathol 2013;41(10):926-7.

5. Fawzi AA, Vo B, Kriwanek R, et al. Asteroid hyalosis in an autopsy population: The University of California at Los Angeles (UCLA) experience. Arch Opbthalmol 2005; 123(4):486-90.

6. Moss SE, Klein R, Klein BE. Asteroid hyalosis in a population: the Beaver Dam eye study. Am 7 Ophthalmol 2001;132(1):70-5.

7. McAlinden C, Skiadaresi E. Asteroid hyalosis. Mayo Clin Proc 2015;90(7):992.

8. Yazar Z, Hanioglu S, Karakoç G, et al. Asteroid hyalosis. Eur 7 Ophthalmol 2001;11(1):57-61.

9. Feist RM, Morris RE, Witherspoon CD, et al. Vitrectomy in asteroid hyalosis. Retina 1990;10(3):173-7.

10. Kachewar SG, Kulkarni DS. An imaging review of intra-ocular calcifications. 7 Clin Diagn Res 2014;8(1):203-5. 